\title{
Aménagement de l'estuaire de la Loire
}

\author{
par J.-F. Maquet \\ Directeur des Accès au Port Autonome \\ de Nantes - Saint-Nazaire
}

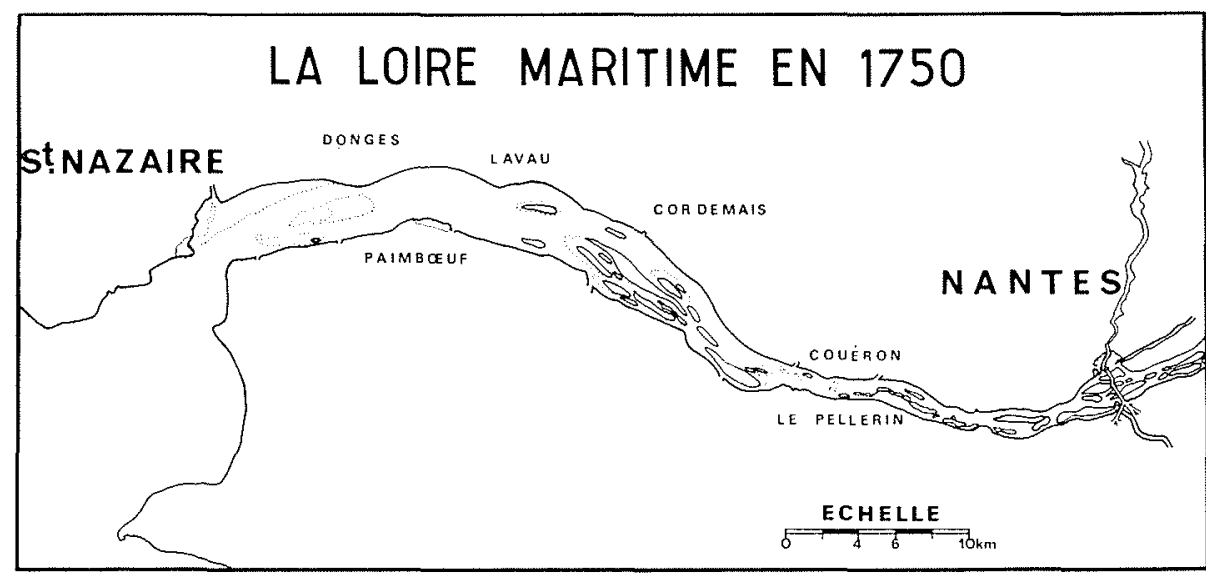

$1 /$

Les grands travaux d'aménagement de la Loire-Maritime ont permis de donner à la navigation un chenal dont le tracé est relativement stable et dont les profondeurs se maintiennent moyennant des dragages d'entretien raisonnables.

Ne bénéficiant pas, comme dans les autres estuaires, des enseignements d'une étude systématique sur modèle réduit, il nous a paru intéressant, dans le cadre d'une session consacrée à l'influence des aménagements sur les équilibres dynamiques dans les estuaires, de montrer comment les ingénieurs ont aménagé la Loire pour la navigation maritime et comment la Loire a réagi à leurs actions ; nous présenterons ainsi dans une première partie les grandes étapes de l'amélioration de l'estuaire.

D'autre part, les observations accumulées au cours des années permettent de mieux connaître certains phénomènes en pertuelle évolution. Nous examinerons donc, dans une deuxième partie, les mouvements de sédiments et la propagation de la marée en Loire.

\section{1 - Les grandes étapes de l'amélioration de l'estuaire de la Loire}

\section{1 - Généralités}

(Fig. 1.)

Pendant des siècles, la Loire Maritime est restée un estuaire naturel étendant ses sinuosités à travers de multiples îles, bancs et faux bras. L'aménagement n'a commencé qu'au dix-huitième siècle lorsque Magin, Ingénieur de la Marine, fut chargé d'exécuter les premiers travaux de digues et d'épis. Jusqu'à la fin du dix-neuvième siècle on s'est surtout préoccupé de rétrécir le lit pour l'approfondir et les endiguements réalisés sur $15 \mathrm{~km}$, de Nantes à la Martinière, ont donné à cette partie du fleuve le nom de «Section endiguée ». 
Au début du vingtième siècle, on a appliqué les idées émises dès 1870 par les Ingénieurs Lechalas et Partiot qui avaient compris le rôle joué par la marée; il en est résulté l'aménagement de la section, dite "Intermédiaire", qui s'étend sur $22 \mathrm{~km}$ de la Martinière à Paimbœuf.

En 1940, un nouveau chenal a été ouvert dans la « Section aval ", entre Paimbœuf et Saint-Nazaire, mettant un terme à l'instabilité chronique de cette région de l'estuaire.

De Saint-Nazaire à la mer, le "Chenal extérieur " a été approfondi dès 1890 pour les besoins du port de SaintNazaire, mais c'est surtout depuis 1960 que de grands travaux sont poursuivis, dans cette section, pour la réception, à Donges, des pétroliers de la classe des $250000 \mathrm{t}$ de port en lourd.

Telles sont les divisions de la Loire en sections aui soulignent les grandes étapes de l'aménagement que nous allons examiner maintenant plus en détail (fig. 2)

\section{2 - Les endiguements}

(Fig. 3.)

\subsection{1 - Travaux de Magin (1756-1768)}

La question de l'amélioration de la Loire Maritime a préoccupé les commerçants nantais depuis le dix-huitième siècle. Dès 1756, Magin s'attaque aux plus mauvaises passes, des barrages submersibles en enrochements sont établis dans les bras secondaires, devant l'île d'Indret et l'île Cheviré, pour réunir les eaux d'étiage en un chenal unique, des épis et des plantations sont réalisés pour favoriser le dépôt des alluvions.

Après des améliorations locales de courte durée, la situation devient aussi mauvaise qu'avant, avec des seuils n'offrant qu'un mouillage de $2 \mathrm{~m}$ en haute mer de morte eau.

\subsection{2 - Travaux de Lemierre (1834-1840)}

Lemierre cherche à resserrer le lit pour que le fleuve exerce un effet de chasse, il construit des digues longitudinales arasées à la cote des hautes mers de morte eau, généralement sur une seule rive, tracées de manière à réduire la largeur du chenal à $200 \mathrm{~m}$ vers Nantes et à $300 \mathrm{~m}$ vers le Pellerin.

Cette première tentative, trop fragmentaire eut des résultats peu sensibles : on avait gagné $0,30 \mathrm{~m}$ sur les seuils, mais de nouveaux atterrissements s'étaient formés.

Les premiers dragages d'entretien entrepris dès 1838 , pendant les périodes de basses-eaux, maintenaient une situation acceptable jusqu'aux crues de l'hiver suivant qui venaient détruire tout le travail de chaque campagne.

\subsection{3 - Travaux de Jegou (1859-1864)}

Ensuite, les ingénieurs ont cherché à réaliser un endiguement continu de Nantes à Paimbœuf, en appliquant la méthode de régularisation par resserrement. Les digues longitudinales n'ont été exécutées que jusqu'à la Martinière, les espaces entre les digues et les anciennes berges étaient laissés libres pour emmagasiner la marée et produire des chasses au jusant.

Ces espaces se sont colmatés très rapidement au fur et à mesure de l'avancement des digues, mais celles-ci ont donné des résultats satisfaisants, dans la Section endi- guée, avec un gain de $1 \mathrm{~m}$ sur les profondeurs. A l'aval des digues, la situation s'est trouvée très aggravée, les alluvions de la partie endiguée se sont déposés dans la section. intermédiaire en formant des atterrissements tels que cette section, qui offrait toujours des tirants d'eau suffisants, a commencé à donner de vives inquiétudes.

\section{3 - L'aménagement de la Section intermédiaire} (Fig. 4)

\subsection{1 - Le projet Lechalas}

L'amélioration de la section intermédiaire fut mise à l'étude en 1867. Deux grands projets très différents furení proposés, l'un de canal latéral, l'autre d'aménagement du fleuve. Ce dernier fut confié aux Ingénieurs Lechalas et Partiot. Véritable précurseur, Lechalas proposait les moyens suivants :

- rescinder toutes les îles qui encombrent la Loire en réduisant celle-ci à un bras unique, régulièrement calibré par des digues, présentant la forme d'entonnoir largement ouvert sur la mer, de façon à faciliter l'introduction du flot;

- creuser en même temps ce lit unique, de manière à abaisser de $2 \mathrm{~m}$ au moins le niveau d'étiage à Nantes, ce qui assurerait l'entretien d'un chenal offrant un tirant d'eau augmenté de $2 \mathrm{~m}$;

- prolonger l'endiguement au-dessus de Nantes jusqu'à la limite amont de la marée.

Ce projet fut jugé trop incertain et trop dispendieux à l'époque et la construction d'un canal latéral de $15 \mathrm{~km}$ fut entreprise en 1882 pour éviter les hauts fonds de la section intermédiaire.

\subsection{2 - Les dragages, le canal maritime (1867-1910)}

Avant la mise en service du canal, des dragages furent effectués de 1867 à 1871 pour maintenir le mouillage de $3,30 \mathrm{~m}$ en haute mer de vive eau. Il fallut, à partir de 1877, augmenter considérablement leur importance pour permettre un gain de $0,50 \mathrm{~m}$ faisant passer le mouillage à $3,80 \mathrm{~m}$ en 1891 .

Le canal fut inauguré en 1892, des dragages à l'amont et à l'aval du canal permirent d'atteindre un mouillage de $5,50 \mathrm{~m}$ en 1898

L'insuffisance du canal apparut rapidement, le tirant d'eau des navires augmentait sans cesse et, dès 1900, les études de Lechalas étaient reprises en vue d'amener l'accès des navires de 6 à $8 \mathrm{~m}$ de tirant d'eau.

En fait, la fréquentation du canal cessa complètement vers 1910 à partir du moment où l'aménagement de la Section intermédiaire, approuvé par une loi du 24 décembre 1903, fut suffisamment avancé.

\subsection{3 - Les travaux de la loi de 1903}

S'inspirant dans les grandes lignes du projet LechalasPartiot, le programme de 1903 comportait une transformation complète de la Section intermédiaire et des parachèvements dans la Section endiguée.

De la Martinière à Paimbœuf, il a fallu remplacer un chenal tortueux, serpentant au milieu d'îles de formes quelconques, par un chenal ayant un tracé rationnel. Les tra- 


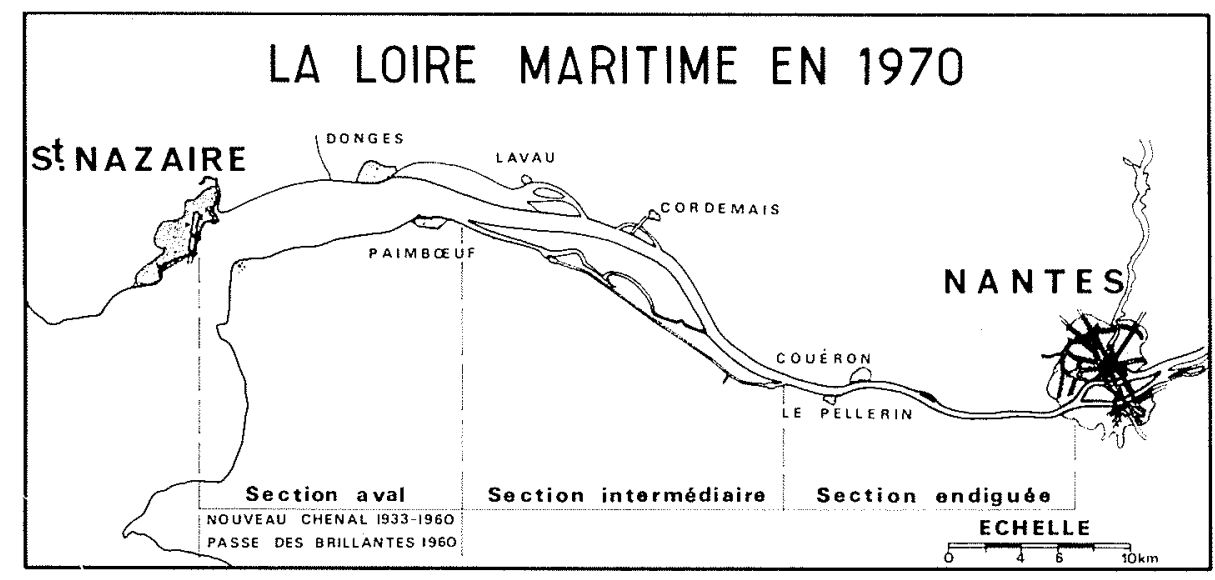

2/

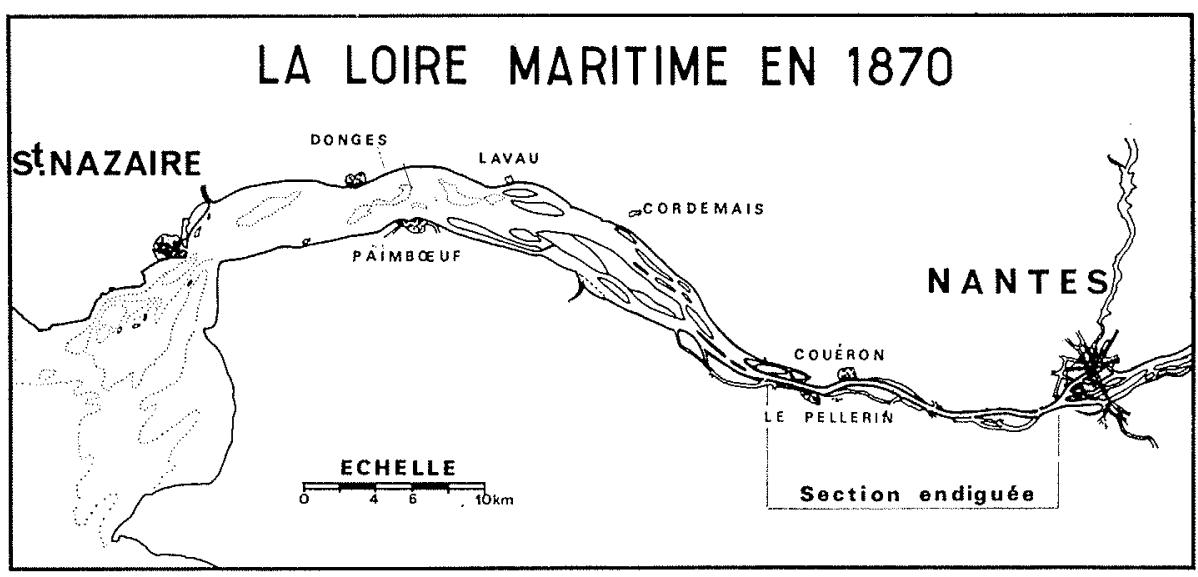

$3 /$

\section{LA LOIRE MARITIME EN 1920}

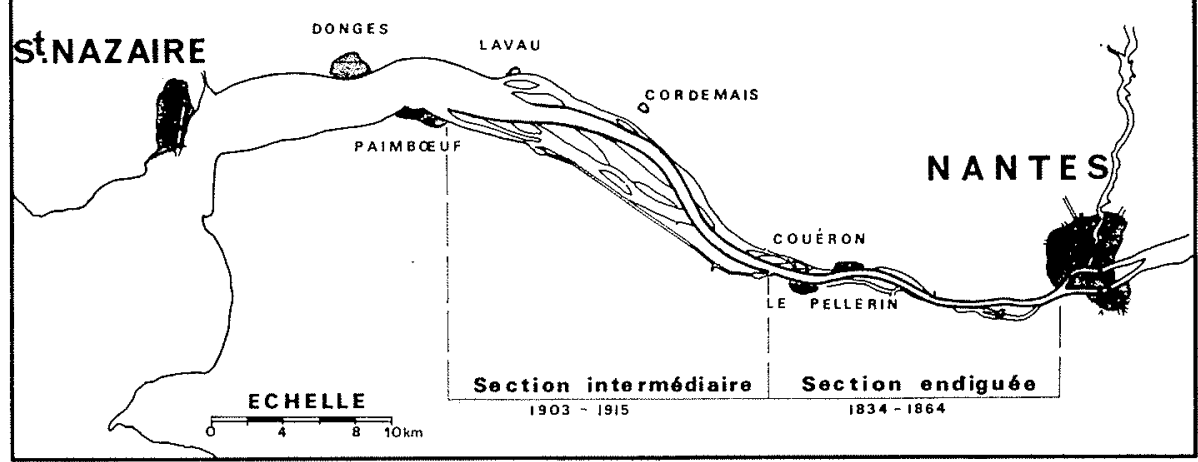




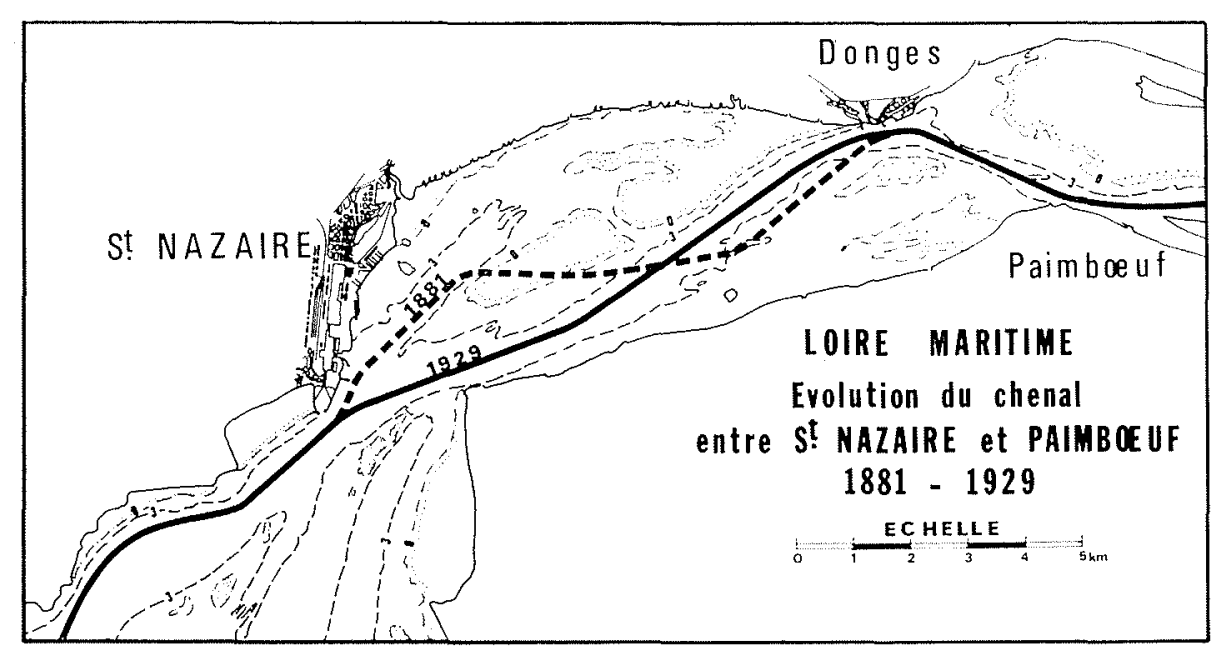

5/

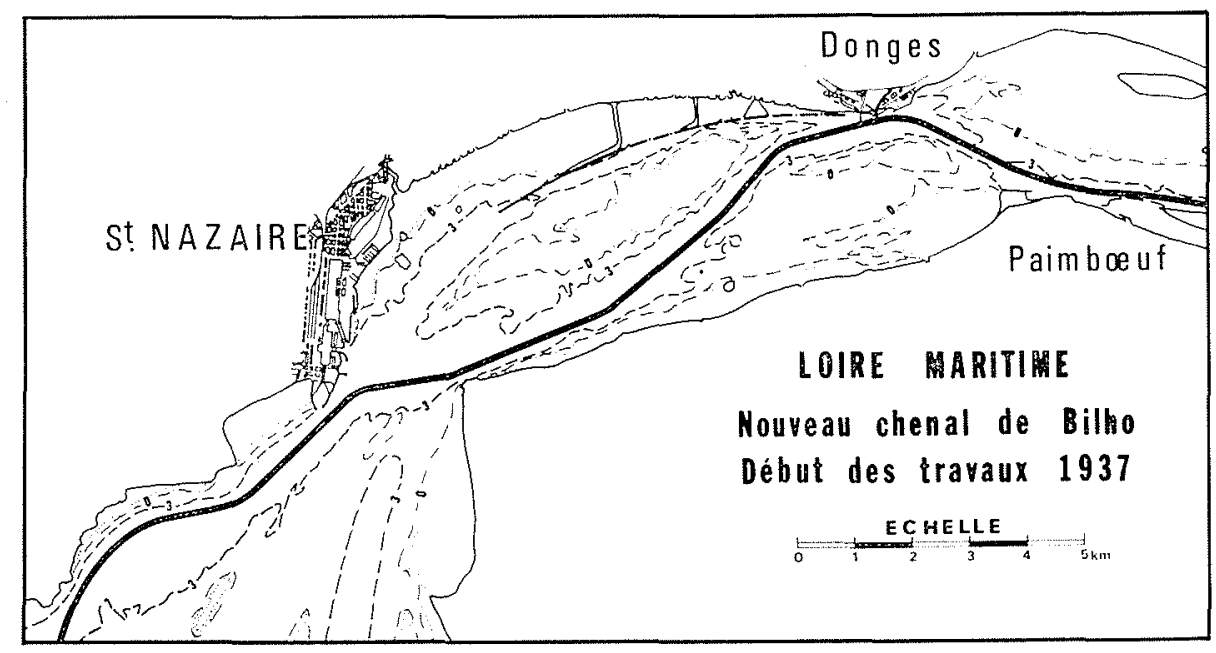

6/

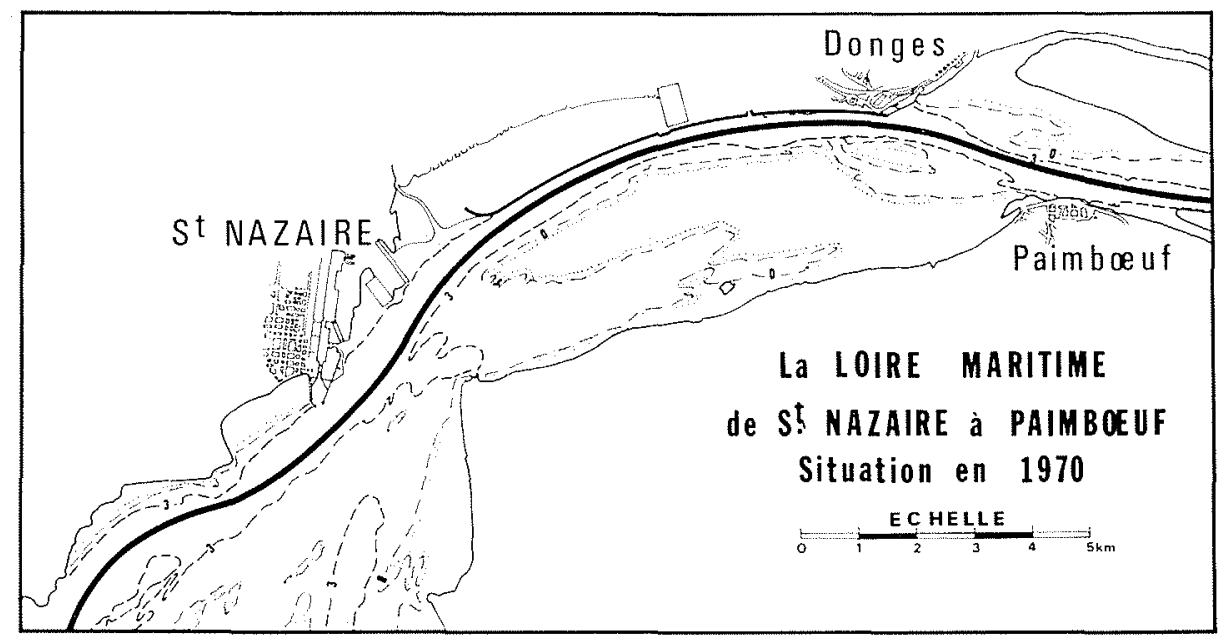

7/ 
vaux comportaient des endiguements continus avec une largeur croissant de $300 \mathrm{~m}$ à $900 \mathrm{~m}$, des rescindements et dragages d'îles (Binet, Grand-Pineau), des dragages d'amélioration pour réduire le travail du fleuve.

Les travaux de la Section endiguée comprenaient une régularisation des formes par épis, déroctages, rescindement ou suppression d'anciennes digues réduisant par trop les sections d'écoulement.

Les résultats de cet aménagement furent très satisfaisants puisque les seuils les plus élevés, qui étaient à la cote $+0,50 \mathrm{CM}$ en 1900 , furent abaissés à $-0,50$ en 1906 , $-1,50$ en $1908,-2,50$ en 1912

Mais ce n'était pas tout de permettre à la marée d'agir efficacement dans la Section intermédiaire, il fallait compléter ces travaux par l'aménagement de la section située à l'amont de Nantes intéressée par la propagation de la marée.

\section{4 - La traversée du port de Nantes ef le Rassin de Marée}

\subsection{1 - Le Bassin de Marée (loi de 1913)}

En 1906 et 1907, deux années sèches pour la Loire, le port de Nantes fut envahi par les vases. Pour remédier aux graves inconvénients qui en résultaient, on avait d'abord envisagé la transformation du port en un vaste bassin à flot à l'aide de barrages, mais le projet était très coûteux et présentait de nombreux aléas du point de vue technique.

Aussi le programme de la loi du 7 juillet 1913 comportait-il l'aménagement de la Loire à l'amont de Nantes. Le but des travaux était d'accroître le volume de flot emmagasiné à chaque marée, de façon à augmenter la vitesse du courant de jusant à une valeur suffisante pour empêcher les vases amenées par le flot de se déposer.

La rectification du fleuve comportait notamment des dragages et la matérialisation du lit mineur par des digues horizontales ou des ouvrages inclinés. Les largeurs étaient croissantes de l'amont à l'aval, le tracé du lit mineur suivait les lois de Fargue.

Cet aménagement a sans doute diminué l'envasement dans le port de Nantes bien qu'il soit difficile d'en juger puisque la nature ne reproduit pas à la demande les conditions de débit fluvial et de coefficient de marée désirées. Ce qui est en tout cas certain c'est que le volume d'eau refoulé par la marée en amont du port de Nantes a augmenté de 6 millions de $\mathrm{m}^{3}$ en vive eau, soit un doublement, les vitesses de jusant qui étaient inférieures à $0,10 \mathrm{~m} / \mathrm{s}$ atteignaient après aménagement $0,25 \mathrm{~m} / \mathrm{s}$ en morte eau exceptionnelle.

Il y a donc eu un progrès important, encore est-il intéressant 'de souligner qu'il ne 's'est réellement affirmé qu'à partir du moment où les radiers des vieux ponts de Nantes ont été démolis (1926 à 1928).

\subsection{2 - La traversée du port de Nantes}

Vers 1925, l'abaissement de l'étiage dans le port de Nantes entraînait l'assèchement de bras secondaires à chaque basse-mer, ce qui présentait des inconvénients pour l'hygiène et pour la tenue des ouvrages avoisinants.

C'est ainsi que furent comblés les bras de la Bourse et de l'Hôpital, en même temps qu'étaient creusés et régularisés les deux bras conservés, de la Madeleine et de Pirmil.

\section{5 - L'aménagement de la Section aval}

\subsection{1 - Le nouveau chenal entre Saint-Nazaire et Donges (1933-1940)}

En 1915, alors que l'aménagement de la Section intermédiaire ouvrait de nouvelles perspectives, on se posait la question de savoir comment il serait possible d'approfondir le chenal entre Paimbœuf et Saint-Nazaire. En 1917, l'établissement maritime de Donges était créé, on ne s'inquiétait pas encore des profondeurs mais surtout de l'instabilité du chenal (fig. 5).

Dans la Section aval, le chenal empruntait d'abord une passe traversière de Paimbœuf à Donges, puis faisait un coude brusque pour aller rejoindre la rive Sud, il traversait ensuite le goulet en changeant de rive une deuxième fois, passant devant Saint-Nazaire et s'infléchissant à nouveau pour prendre la route du large (fig. 6).

La solution adoptée, à la suite des études poursuivies au cours des années 1926 à 1928, a consisté à substituer, au tracé défectueux et instable, un nouveau tracé à concavité unique, reliant Donges à Saint-Nazaire suivant une courbure appuyée sur la rive Nord et se raccordant avec l'inflexion des Brillantes entre Donges et Paimbœuf. Les travaux, déclarés d'utilité publique en 1933, aboutirent, en 1940, à l'ouverture du nouveau chenal, dit de "Bilho", le long de la digue de "Montoir" (fig. 7).

Dans l'ensemble, on peut dire que ce programme très audacieux a été un succès éclatant, puisque le tracé s'est parfaitement maintenu le long de la digue de Montoir et que le chenal a pu être approfondi à $3 \mathrm{~m}$, élargi de $180 \mathrm{~m}$, sans difficulté particulière pour l'entretien.

Le régime hydraulique n'a pas accusé de modification substantielle, le nouveau chenal était un sillon de section insuffisante pour modifier le régime de propagation de la marée.

La digue a été construite à $1 \mathrm{~m}$ au-dessus du niveau des hautes mers de vive eau exceptionnelles et reliée à la rive par des épis. L'espace entre la digue et l'ancienne rive s'est rapidement colmaté.

\subsection{2 - Rectification de la passe des Brillantes (1960-1961)}

On pensait que l'ouverture du nouveau chenal modifierait les courants dans la région de Paimbœuf de manière que l'inflexion des Brillantes prenne un caractère moins traversier. Après la guerre, on constatait que la passe, au lieu de se redresser, avait légèrement pivoté de façon à se trouver plus traversière.

Aussi, en 1948, furent décidés des essais sur modèle réduit qui furent réalisés de 1949 à 1956.

On a recherché une modification de l'inflexion des Bril. lantes pour lui donner une plus grande stabilité. Les travaux, exécutés en 1960 et 1961 , ont réalisé un premier déplacement de la passe.

La présence d'une plature rocheuse avait conduit à un déplacement plus limité que ne le préconisait le laboratoire. Cependant, les résultats ont été assez satisfaisants, 
bien que la concordance des actions du flot et du jusant ne soit pas encore parfaite.

Des sondages ayant permis de mieux délimiter la plature rocheuse, une nouvelle rectification de la passe des Brillantes a été entreprise, en 1973, pour éviter le seuil rocheux et tenter d'assurer encore un meilleur raccordement avec le chenal de Bilho.

\section{6 - Le Chenal extérieur}

(Fig. 8.)

\subsection{1 - Ouverture de la passe des Charpentiers (1890-1913)}

Le Chenal extérieur comporte, à partir de Saint-Nazaire, une partie courbe qui suit la côte, c'est la courbe de «Bonne Anse», et une partie rectiligne qui emprunte un sillon creusé à l'origine par les courants et traverse le hautfond de la passe dite "des Charpentiers».

$\mathrm{Au}$ dix-huitième siècle, des profondeurs de $5 \mathrm{~m}$ sous basse-mer existaient sur le seuil, les passes d'accès à la Loire présentaient alors des conditions éminemment favorables à la navigation. Ces profondeurs étaient largement suffisantes compte tenu des vicissitudes que connaissait le chenal de navigation entre Nantes et Saint-Nazaire.

Mais ces mêmes vicissitudes avaient conduit à la création du port de Saint-Nazaire. Un premier bassin avait été ouvert en 1856 et un deuxième en 1881. Parallèlement, on avait constaté, de 1864 à 1881 , un relèvement de $80 \mathrm{~cm}$ de la passe des Charpentiers, il fallait donc songer à l'aménager afin qu'elle ne soit pas un obstacle pour l'accès à Saint-Nazaire.

Un sillon d'essai, creusé en 1890, avait permis un approfondissement de 25 à $50 \mathrm{~cm}$, qui s'était maintenu pendant une année. On décida, en 1892, d'entreprendre le creusement, à titre définitif, d'un chenal de $100 \mathrm{~m}$ de largeur à la cote $-5 \mathrm{~m}$. Des dragages d'entretien, d'environ $250000 \mathrm{~m}^{3}$ par an, permirent de conserver la cote moyenne de $-5,50$, les fonds se relevaient pendant l'hiver et les campagnes annuelles de dragage rétablissaient la situation.

En 1906, l'ouverture d'une nouvelle entrée aux Bassins de Saint-Nazaire offrait des possibilités accrues à la navigation et rendait nécessaire un approfondissement du chenal extérieur pour profiter pleinement 'des améliorations obtenues.

Un approfondissement à la cote -7 , sur $200 \mathrm{~m}$ de large, fut réalisé par une drague à godets. Cette cote a été entretenue jusqu'en 1935 moyennant l'exécution de dragages d'entretien d'environ $250000 \mathrm{~m}^{3}$ par an. On avait à la cote -7 , comme à la cote -5 , un chenal stable dont l'entretien était relativement facile.

\subsection{2 - Sortie du « Normandie " et nouvel approfondissement (1934-1942)}

En 1934, pour la sortie du paquebot Normandie construit à Saint-Nazaire, un dragage intensif de $2400000 \mathrm{~m}^{3}$ était entrepris pour donner la cote -8 sur $150 \mathrm{~m}$. Ce sillon étroit, creusé pour le passage d'un navire exceptionnel, ne devait pas se maintenir et, de 1935 à 1938, on constatait des apports annuels de $600000 \mathrm{~m}^{3}$ sur la passe.

Mais, de 1939 à 1942, l'extraction de $2600000 \mathrm{~m}^{3}$ pour atteindre la cote $(-8,5)$ sur une passe plus large, donnait de bien meilleurs résultats. Bien qu'il n'y ait pas eu de travaux de 1942 à 1947, la passe ne s'est pas comblée et les apports annuels, dragués en entretien, ont varié entre 250000 et $300000 \mathrm{~m}^{3}$.

Ceci met en évidence d'une part l'importance d'une opération massive avec des engins puissants, et d'autre part, le fait que le chenal extérieur dragué à la cote $-8,50$ ne nécessite pas plus d'entretien que lorsqu'il était dragué à la cote -7 ou à la cote $-5,50$.

Dans cette vue d'ensemble des diverses phases de l'amélioration du fleuve dans sa partie maritime, nous avons pu vérifier combien chaque section est solidaire de celles qui l'entourent et à quel point la continuité d'un aménagement est nécessaire à son succès. Il est intéressant maintenant d'examiner quelques aspects particuliers du régime du fleuve dont l'évolution peut être liée plus ou moins directement aux actions de l'homme sur la nature.

\section{2 - Evolution de phénomènes particuliers}

\section{1 - Mouvements de sédiments}

\subsection{1 - Dragages d'entretien des chenaux}

Depuis Porigine des travaux, le chenal de navigation a été déplacé, approfondi de plus de $5 \mathrm{~m}$ sur les seuils, élargi de près de $150 \mathrm{~m}$ dans la partie aval, la question peut être posée de savoir si ces améliorations n'ont pas eu pour conséquence une augmentation excessive des dragages d'entretien. On peut estimer à $1500000 \mathrm{~m}^{3}$ le volume moyen des dragages d'entretien annuels, effectués au début du siècle, dans les chenaux d'accès depuis la mer jusqu'à Nantes.

Vers 1940, le volume des dragages d'entretien annuels restait du même ordre de grandeur avec $1400000 \mathrm{~m}^{3}$. De 1960 à 1970, la moyenne des dragages d'entretien est de $400000 \mathrm{~m}^{3}$ de la mer jusqu'à Donges et de $900000 \mathrm{~m}^{3}$ de Donges à Nantes, soit au total $1300000 \mathrm{~m}^{3}$.

Ces moyennes cachent, bien entendu, de grandes inégalités entre les années sèches et les années humides, et des changements dans la nature et dans l'emplacement des dépôts, mais il est quand même intéressant de constater que le volume des dragages d'entretien des chenaux n'a pratiquement pas changé malgré les importantes modifications du chenal.

Toutefois, cette situation évolue au cours des dernières années; en effet, les caractéristiques des navires modernes ont nécessité la création de rades de grandes dimensions qui introduisent une discontinuité dans les sections et favorisent les dépôts de matériaux meubles. D'autre part, les exigences d'une navigation plus intense conduisent à des interventions plus fréquentes et il en résulte une augmentation sensible des dragages d'entretien dans les chenaux.

\subsection{2 - Mouvements de sable : Les grandes rides (Fig. 9.)}

En 1919, alors que les travaux de la Section intermédiaire avaient permis un approfondissement notable, les 


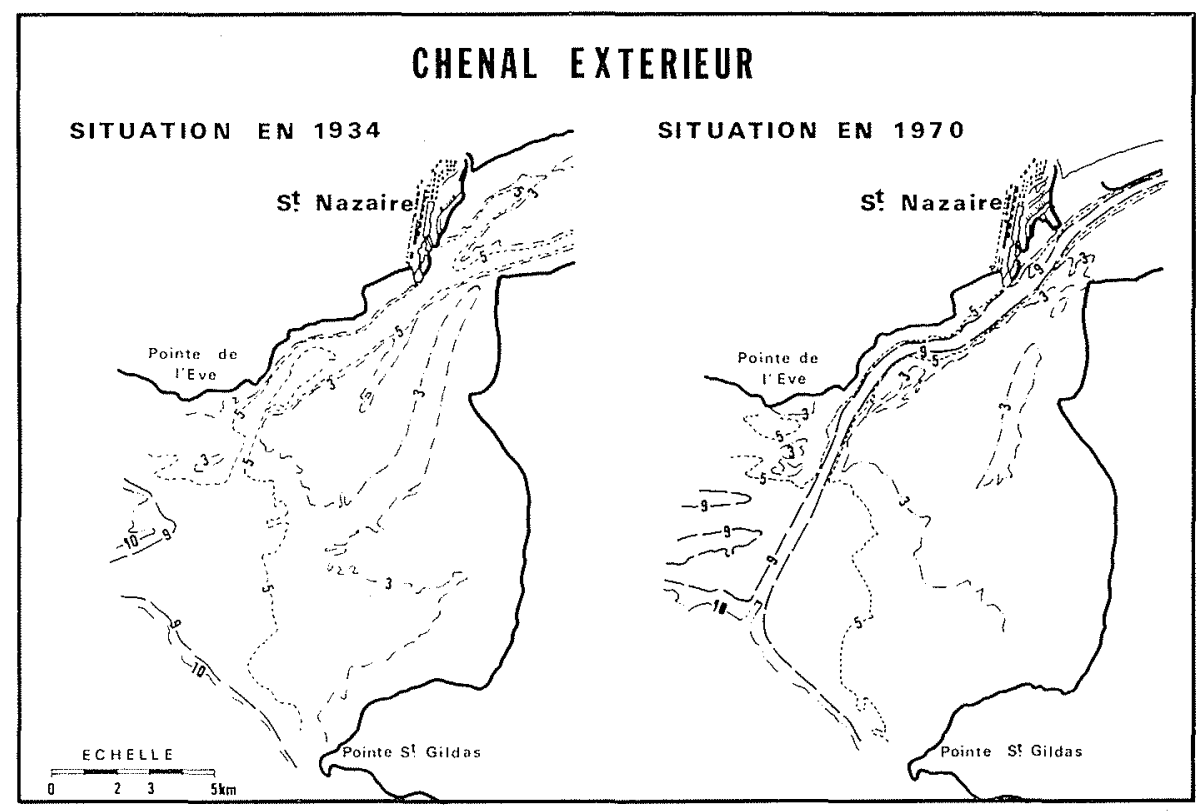

8/

LOIRE MARITIME

SONDAGES EFFECTUES DANS LA SECTION INTERMEDIAIRE

SUR L'AXE DU CHENAL DE NAVIGATION

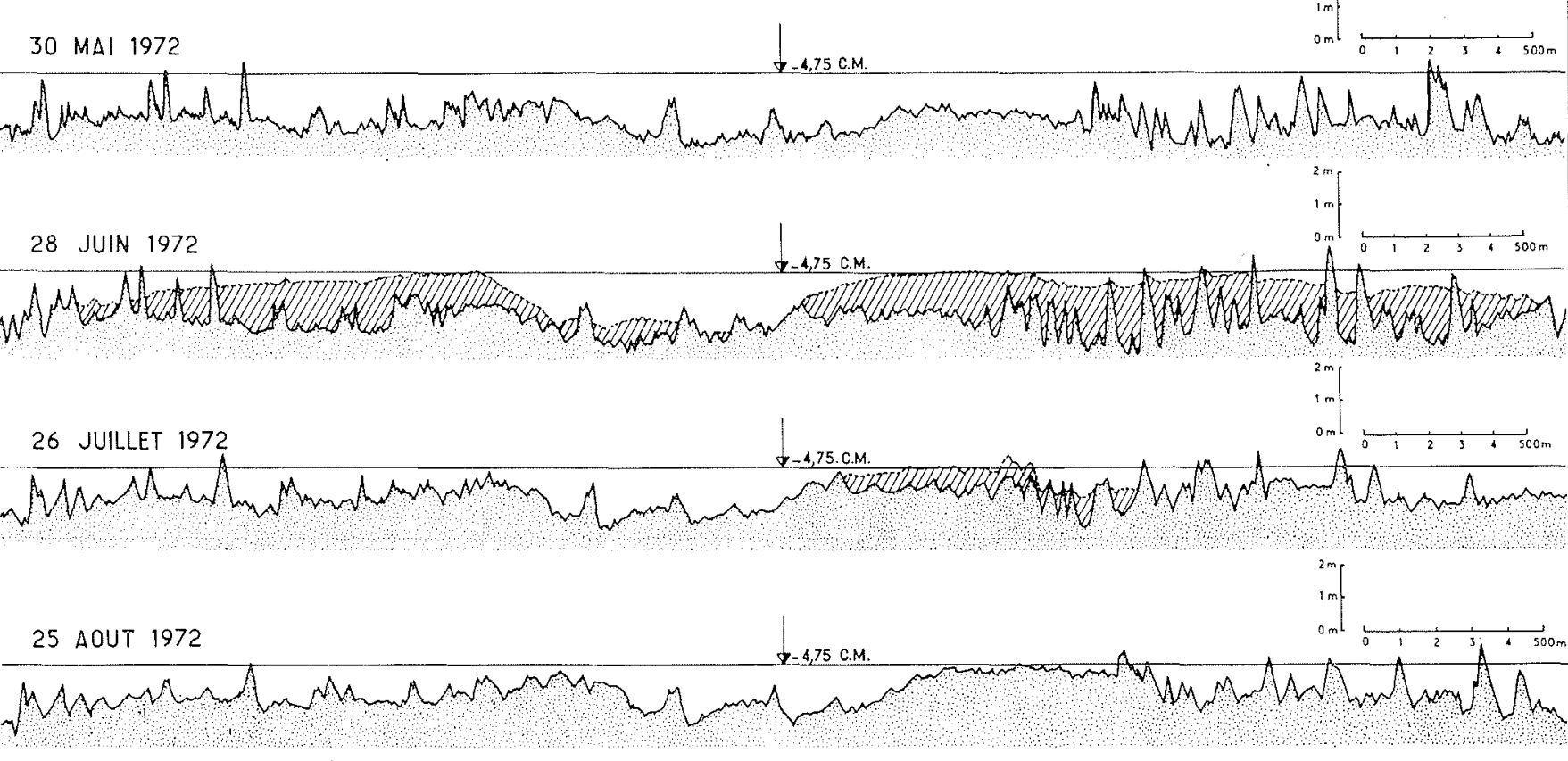


ingénieurs constataient l'existence de «champignons» de sable faisant saillie de $1 \mathrm{~m}$ à $1,50 \mathrm{~m}$ en divers points du chenal. En 1937, l'acquisition d'un sondeur à ultra-sons permettait de mieux connaitre "les grandes rides» qui déforment les fonds tapissés de sable et limitent le tirant d'eau offert à la navigation. Ce phénomène a fait l'objet d'une étude approfondie par M. Ballade.

Les grandes rides se présentent comme des ondulations dont l'amplitude moyenne est de $1,50 \mathrm{~m}$ et la « longueur d'onde " une centaine de mètres. En période d'étiage de la Loire, la croissance des déformations est particulièrement accentuée et il n'est pas rare de relever des dénivellations de 2 à $3 \mathrm{~m}$; les crêtes sont relativement stables et se reforment après dragage. Lorsque le débit d'amont croît, les rides cheminent vers l'aval en se déformant, elles sont écrétées par les crues.

On peut dire que l'aménagement de la Loire n'influence pas directement le phénomène, lorsque le chenal est approfondi, l'amplitude des rides se conserve, l'abaissement général se répercute sur la cote des crêtes mais celles-ci repoussent avec autant de vigueur.

Il faut cependant préciser que, dans certaines zones, le dragage continuel finira par diminuer le stock de sable susceptible de reformer des rides puisque celui-ci est de moins en moins réalimenté par l'amont.

Lechalas notait déjà, en 1869: "Le volume des sables qui descend chaque année les vals de la Loire et de l'Allier est de $1000000 \mathrm{~m}^{3} ; 600000 \mathrm{~m}^{3}$ sont enlevés par les riverains, les $400000 \mathrm{~m}^{3}$ restants se partagent entre les dépôts sur les grèves de l'embouchure et l'écoulement à la mer. Une civilisation plus intense suffirait pour débarrasser la Loire des $400000 \mathrm{~m}^{3}$ formant la fâcheuse balance qui lui incombe, car des populations plus denses et plus riches bâtiraient davantage, sableraient une plus grande surface de jardins».
En 1941, M. l'Ingénieur en Chef Gibert estimait le volume des apports de la Loire fluviale, en aval d'Angers, à un minimum de $1200000 \mathrm{~m}^{3}$, dont $300000 \mathrm{~m}^{3}$ étaient extraits par les entreprises d'extraction de sable pour les besoins des riverains. A partir de 1949, on constate une diminution des dépôts de sable dans le port comme conséquence de l'augmentation du volume des dragages des entreprises en amont.

En 1964, le cube enlevé par les sabliers, entre Nantes et Angers, était de $1500000 \mathrm{~m}^{3}$, en 1972, il dépassait $2000000 \mathrm{~m}^{3}$. On comprend, dans ces conditions, qu'on ne drague plus de sable dans le port de Nantes et que les importantes fosses qui existent ne soient plus comblées par les crues ordinaires.

\subsection{3 - Mouvements de vase : Le bouchon vaseux}

Le transport en suspension en Loire Maritime, pour être moins spectaculaire que dans certains estuaires comme celui de la Gironde, n'en est pas moins extrêmement net et a appelé depuis longtemps l'attention des ingénieurs. Pendant certaines périodes et par endroit, on observe des eaux extrêmement chargées en vase qui constituent ce qu'on appelle le bouchon vaseux. Ce phénomène, commun à plusieurs estuaires, a été étudié en Loire par M. L. Berthois et, tout dernièrement, par M. B. Gallenne, du Laboratoire de Géologie Marine de Nantes.

La diversité des facteurs qui entrent en jeu, et la difficulté de les mesurer simultanément en plusieurs endroits, ne nous permettent pas de déterminer complètement l'influence des aménagements sur l'évolution de ce phénomène. Bien sûr, on peut penser qu'avec l'approfondissement des accès portuaires, la limite amont du bouchon vaseux recule en même temps que la limite de propagation de la marée.

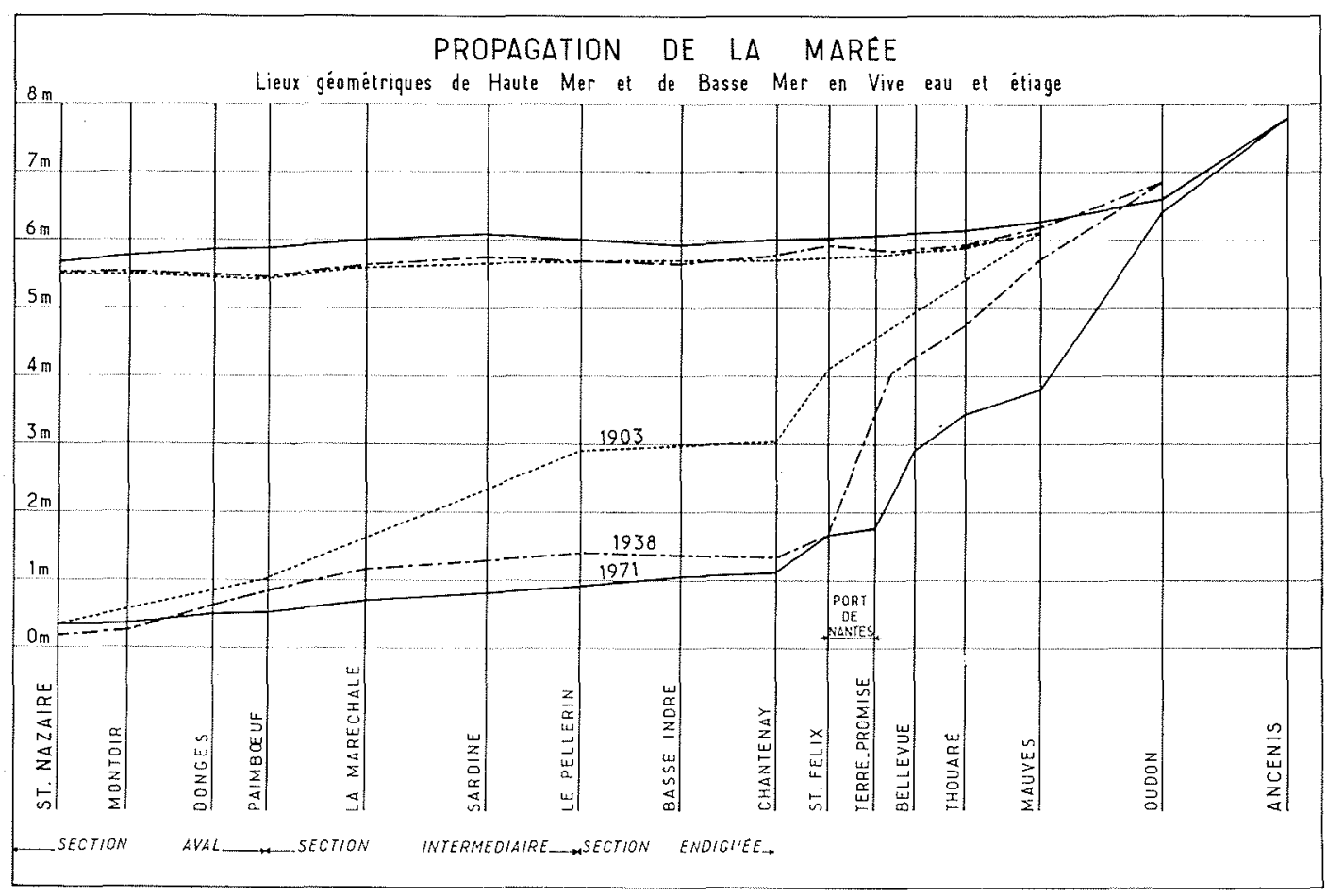


mais l'envasement du port de Nantes, en année sèche, ne date pas d'aujourd'hui et il est certain que le débit fluvial exerce une influence très importante. On sait également que la création de zones élargies pour l'évolution des grands navires favorise la sédimentation, mais cela est tout à fait normal et n'ajoute rien à la connaissance du phénomène. M. Berthois a formulé, en 1968, une théorie de la formation du bouchon vaseux en Loire.

Les crues de la Loire, principalement celles d'automne qui balaient les zones du fleuve exondées depuis plusieurs mois, et celles de printemps, qui succèdent aux labours du mois de mars qui ont ameubli le sol en favorisant l'érosion par ruissellement, entraînent des vases en suspension jusqu'au large de la Loire où elles se déposent. $\mathrm{Au}$ cours des grandes marées de fin de printemps, ces vases sont reprises et viennent réalimenter le bouchon vaseux à une époque où le débit fluvial n'opposera pas la même résistance qu'en hiver.

On connaît également la position approximative du bouchon vaseux en fonction du débit fluvial et du coefficient de marée.

Les récentes études effectuées par M. Gallenne, avec le concours du Port Autonome de Nantes - Saint-Nazaire et du C.N.E.X.O., ont permis de distinguer les rôles respectifs du bouchon vaseux, zone de turbidité élevée (de $50 \mathrm{mg} / 1$ à $10-20 \mathrm{~g} / \mathrm{l}$ ), et de la crème de vase, zone dense à très forte concentration (turbidité supérieure à $20 \mathrm{~g} / 1$ ).

La crème de vase s'enrichit, pendant les étales, par décantation des matériaux provenant du bouchon vaseux et celui-ci est réalimenté par la remise en suspension de la crème de vase par les courants.

M. Gallenne a également mis en évidence la présence d'une concentration de Montmorillonite dans les sédiments en suspension et son intérêt comme traceur naturel pour suivre les déplacements du bouchon vaseux et de la couche crème.

Nos connaissances progressent, mais il reste encore beaucoup à faire pour déterminer les quantités de matériaux existant dans le bouchon vaseux et dans quelles proportions celui-ci est alimenté par l'amont ou par l'aval, suivant les tempêtes, les marées, les crues.

\section{2 - Propagation de la marée}

\subsection{1 - Lieux géométriques de haute mer et de basse mer \\ (Fig. 10.)}

L'examen des lieux géométriques de haute mer et de basse mer à diverses époques permet d'avoir une vue d'ensemble de l'état du fleuve et d'apprécier les conséquences des aménagements réalisés.

De 1903 à 1971, on n'observe que peu de modifications du niveau des hautes mers, les conditions atmosphériques pouvant être responsables des légères différences notées entre le lieu géométrique couramment observé aujourd'hui et celui de 1938 ou de 1903, il semble toutefois se dégager une légère tendance à l'augmentation du relèvement vers l'amont.

- En 1938, pour une cote de $(+5,52)$ à Saint-Nazaire, on avait relevé $(+5,75)$ à Sardine, $(+0,23)$ à mi-distance entre Nantes et Saint-Nazaire et $(+5,77)$ à Nantes-Chantenay $(+0,25)$.
- En 1971, pour une cote $(+5,67)$ à Saint-Nazaire, on a relevé $(+6,09)$ à Sardine $(+0,42)$ et $(+6,00)$ à Nantes-Chantenay $(+0,33)$.

Par contre, les lieux géométriques des basses mers se sont beaucoup modifiés.

En premier lieu, le point fluvial, intersection des lieux géométriques des basses mers de vive eau et de morte eau, était à $20 \mathrm{~km}$ en aval de Nantes avant les travaux de la Section intermédiaire. En 1938, il était reporté à la. limite amont du port de Nantes, il se trouve maintenant dans la région de Bellevue, $10 \mathrm{~km}$ plus à l'amont. On remarque, à ce sujet, que la présence d'un seuil rocheux à cet endroit apporte une perturbation certaine à l'écoulement des basses eaux et que la suppression de celui-ci permettrait sans doute de situer le point fluvial légèrement à l'aval de Thouaré, c'est-à-dire qu'il se serait déplacé d'une quinzaine de kilomètres depuis 1938.

La limite de propagation de la marée a été également reculée vers l'amont.

Au début du siècle, cette limite était, en morte eau, à $8 \mathrm{~km}$ à l'amont de Nantes; en 1938, à $12 \mathrm{~km}$; en 1971, elle était à $25 \mathrm{~km}$.

En vive eau, la limite de propagation de la marée se trouve maintenant à Ancenis, $35 \mathrm{~km}$ à l'amont de Nantes, au lieu de $24 \mathrm{~km}$ en 1938 et $15 \mathrm{~km}$ en 1903 .

L'allure générale du lieu géométrique des basses mers de vive eau est très symptomatique des progrès réalisés depuis le début du siècle. En 1903, la pente moyenne du lieu géométrique entre Saint-Nazaire et Nantes-Chantenay était de $5 \mathrm{~cm}$ par $\mathrm{km}$; en 1938, la pente était de $2,23 \mathrm{~cm}$ par $\mathrm{km}$, ce qui dénote l'effet bénéfique des travaux de la section intermédiaire.

En 1954, on note une pente moyenne de $1,7 \mathrm{~cm} / \mathrm{km}$ avec, notamment, un meilleur profil dans la région de Donges-Paimbœuf en raison de la création du nouveau chenal. En 1971, la pente était de $1,55 \mathrm{~cm} / \mathrm{km}$, elle peut être estimée actuellement à environ $1,2 \mathrm{~cm} / \mathrm{km}$. Cette pente moyenne a donc beaucoup diminué, ce qui est le signe d'une amélioration notable des conditions de propagation de la marée.

On a même constaté, en aval de Paimbœuf, lors des marées de vive eau du début d'avril 1973, une contre-pente, l'étiage étant descendu 3 à $6 \mathrm{~cm}$ plus bas que l'étiage à Saint-Nazaire; il est permis de penser que l'aménagement apporté au chenal, entre Saint-Nazaire et Donges, est responsable de cette amélioration du lieu géométrique des basses mers.

\subsection{2 - Cote des basses mers d'étiage à Nantes}

C'est évidemment à Nantes, et à l'amont de ce port, qu'on note les modifications les plus importantes.

En 1876, le marnage en vive eau à Nantes-Chantenay était de $2,09 \mathrm{~m}$, il passait à $3,47 \mathrm{~m}$ en 1909 ; à cette date, en morte eau et en étiage, le courant ne renversait pas dans le port de Nantes. En 1937 , on notait $4,25 \mathrm{~m}$ et, en $1971,4,89 \mathrm{~m}$.

La progression du marnage en vive eau à Nantes -SaintFélix se présente différemment, de $1,65 \mathrm{~m}$ en 1903 , il était de $4,12 \mathrm{~m}$ en 1937 , mais n'est que de $4,35 \mathrm{~m}$ en 1971 .

Ainsi, entre Saint-Félix et Chantenay, distants de $3,850 \mathrm{~km}$, la différence de marnage, qui était très importante $(1,82 \mathrm{~m})$ en 1903 , était réduite à $13 \mathrm{~cm}$ en 1937 après les travaux de la Section intermédiaire et du bassin de 


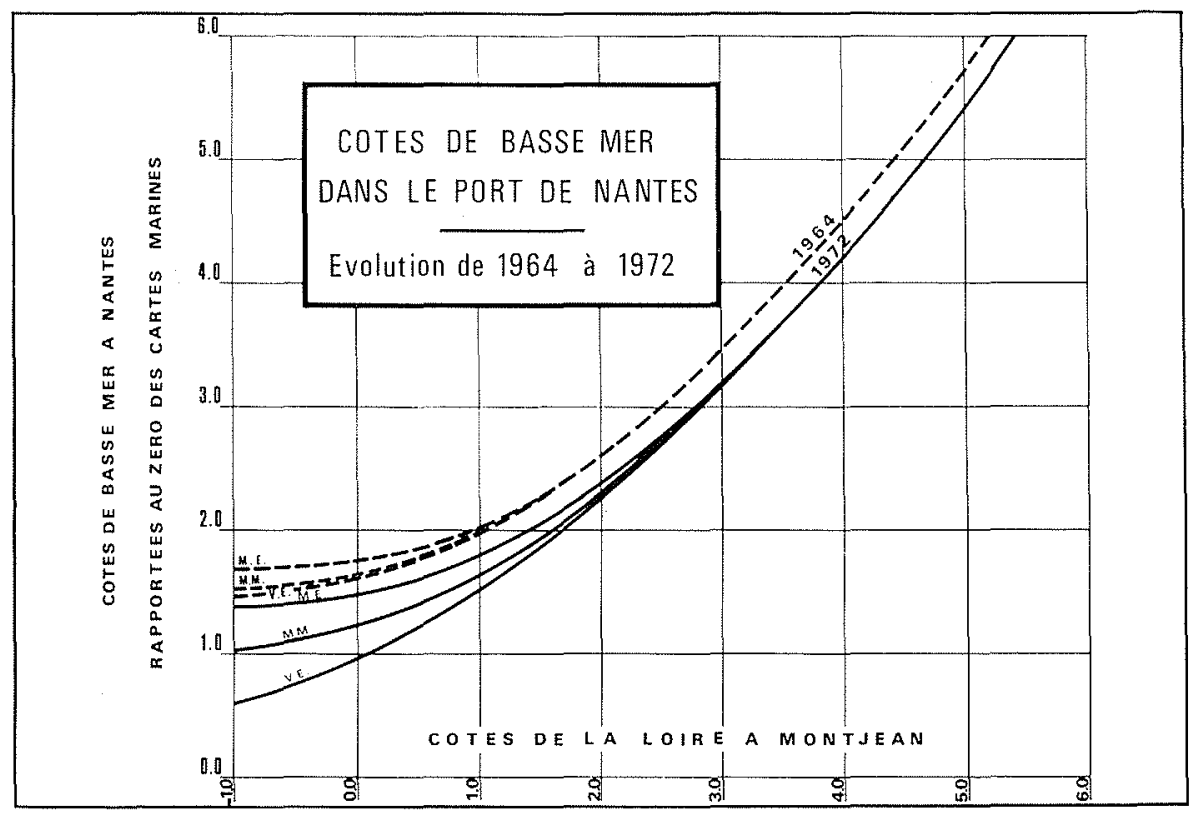

$11 /$

marée. Maintenant, la différence est de $54 \mathrm{~cm}$ entre Chantenay et Saint-Félix parce que les radiers des ponts et les vestiges de la dernière guerre constituent un obstacle inafouillable et introduisent une anomalie dans le lieu géométrique des basses mers.

D'une façon générale, l'augmentation du marnage est due principalement à l'abaissement de la cote des basses mers en étiage à Nantes.

De 1850 à 1900, il y a eu un abaissement d'étiage de l'ordre de $40 \mathrm{~cm}$ consécutif aux travaux effectués dans la section endiguée. Depuis 1900 , on note un abaissement de $1,25 \mathrm{~m}$ jusqu'en 1933 et de $1,57 \mathrm{~m}$ jusqu'en 1938. La cote de $(+1,25 \mathrm{CM})$ a été exceptionnellement constatée à Nantes en 1938 et 1939.

Le niveau des basses mers de vive eau dans le port de Nantes s'est abaissé régulièrement depuis 1938 jusqu'en 1971, année où on a relevé, au marégraphe de Chantenay, une cote de $(+1.11 \mathrm{CM})$ au-dessus du zéro des cartes marines à Saint-Nazaire, pour une marée de coefíicient 95 , un débit fluvial voisin de l'étiage et des conditions atmosphériques normales. Cependant, on assiste actuellement à une accélération de cette évolution qui fait que lon admet que le niveau des basses mers de vive eau, dans le port de Nantes, atteindrait normalement la cote de $(+0,97 \mathrm{CM})$, c'est-à-dire que l'abaissement d'étiage depuis 1938 est de $0,36 \mathrm{~m}$.

La cote mentionnée ci-dessus, de $0,97 \mathrm{CM}$, est une cote moyenne de vive eau, mais la plus basse basse-mer enregistrée à Nantes a été de $(+0,47 \mathrm{CM})$ le 14 juillet 1972 pour un coefficient de marée de 89 , une pression barométrique élevée et un vent favorisant le jusant, alors que le débit fluvial amont était nettement en-dessous de l'étiage du fleuve ( $-0,27 \mathrm{~m}$ à l'échelle de Montjean). Ceci montre que le niveau des basses mers à Nantes est très sensible à l'état du fleuve à l'amont et aux conditions atmosphériques.

Pour préciser ce phénomène, une étude comparative des basses-mers à Nantes, des coefficients de marée et des débits du fleuve a été effectuée de 1964 à 1972. Malgré la dispersion des points, il est possible de tracer des courbes moyennes de la cote à Nantes en fonction du débit à Montjean pour les vives eaux, les marées moyennes et les mortes eaux (fig. 11).

Il n'est pas surprenant de voir que les cotes les plus basses à Nantes correspondent, en moyenne, aux vives eaux et à un faible débit fluvial. Au voisinage de l'étiage de $225 \mathrm{~m}^{3} / \mathrm{s}$, le passage d'une vive eau à une morte eau produit le même relèvement de la cote qu'un doublement du débit fluvial

L'examen de l'évolution de ces courbes moyennes de 1964 à 1972 montre que l'abaissement des cotes de bassemer à Nantes s'est nettement manifesté à partir de 1970. On constate également que l'influence du coefficient de marée, qui ne se faisait sentir à Nantes qu'au-dessous d'un débit fluvial de $700 \mathrm{~m}^{3} / \mathrm{s}$ en 1964 , est encore notable pour un débit de $1500 \mathrm{~m}^{3} / \mathrm{s}$.

L'évolution des cotes de basse-mer dans le port de Nantes n'est sans doute pas terminée, car le régime marin prend le pas sur le régime fluvial.

\section{Conclusion}

L'histoire de l'amélioration de la Loire Maritime est une illustration constante des grands principes de solidarité et de continuité. Nous avons pu, dans certains cas, mettre en évidence les réactions du fleuve aux aménagements successifs, réalisés pour les besoins de la navigation: apparition d'un engraissement dû à une discontinuité, approfondissement favorisé par la concordance des actions du flot et du jusant, recul de la limite de propagation de la marée.

Néanmoins, les aménagements de navigation ne sont pas les seuls et l'évolution de l'estuaire de la Loire est 
influencée par toutes sortes de phénomènes qui se produisent depuis la source jusqu'à l'embouchure. La diminution de l'ensablement dans le port de Nantes résulte de l'augmentation spectaculaire des quantités de sables prélevées en amont pour les besoins de la construction. L'accroissement des besoins en eau du bassin versant et la lutte contre les inondations nécessitent la construction d'ouvrages de régularisation qui modifieront le régime hydraulique du fleuve. Les transports en suspension pourront varier avec la surface des terres cultivées et en fonction de la configuration des rives.
La synthèse de toutes ces influences est bien difficile à faire et l'amélioration d'un fleuve à marée n'en est que plus complexe. L'expérience des années précédentes montre que les possibilités que la Loire peut offrir à la navigation sont loin d'être épuisées. L'évolution importante, constatée ces dernières années, doit donc se poursuivre, mais il serait hasardeux de l'attribuer à la seule action des travaux d'aménagement. Ceux-ci n'étant qu'un des nombreux facteurs qui se composent pour maintenir certains phénomènes dans une tranquilité relative et en précipiter d'autres vers des transformations irréversibles.

\section{Discussion}

Président : M. J. CHAPON

M. le Président remercie M. Maquet de son exposé qui a bien mis en lumière les divers problèmes que pose l'aménagement de l'estuaire de la Loire.

Il ouyre ensuite la discussion en demandant au conférencier

- Quelle est la vitesse de déplacement longitudinal des rides observées sur le fond de la Loire?

- A-t-on une idée des facteurs de leur formation et de leur déplacement?

On a constaté, répond M. MAqueT, qu'une augmentation du débit fluvial entraîne une progression de rides vers l'aval mais $M$. BALLADE, qui a étudié cette question, pourrait fournir des données plus précises.

Les rides, dit $M$. Ballade, sont en général constituées par des sables de Loire grossiers; elles progressent vers laval de quelques dizaines de mètres par an. Une étude déjà ancienne, tendant à déterminer l'évolution de l'amplitude en fonction de la profondeur d'eau, avait conclu que cette amplitude restait la même lorsqu'on approfondissait le chenal.

Les observations faites sur la Loire maritime, dans la région de l'île de Bois, ont montré qu'en période d'étiage, le riden reste dans une position moyenne invariable.

Son cheminement vers laval s'amorce lorsque croît le débit d'amont et s'intensifie en même temps que celui-ci. On à observé les vitesses suivantes :

\begin{tabular}{|c|c|}
\hline Débit Fluvial & Cheminement Journalier \\
\hline $130 \mathrm{~m}^{3} / \mathrm{s}$ & $0 \mathrm{~m}$ \\
$480 \mathrm{~m}^{3} / \mathrm{s}$ & $0,50 \mathrm{~m}$ \\
$1260 \mathrm{~m}^{3} / \mathrm{s}$ & $1,65 \mathrm{~m}$ \\
$2280 \mathrm{~m}^{3} / \mathrm{s}$ & $3,50 \mathrm{~m}$ \\
\hline
\end{tabular}

Ces résultats se rapportent à des observations faites au cours des années 1950,1951 et 1952.
Sur une question de M. Leroy, M. Maquet indique que du fait de la progression des extractions de sable entre Saint-Nazaire et Nantes, il semble aue l'on trouve de moins en moins de sable à l'amont immédiat de Nantes.

La Direction du Bâtiment et des Travaux Publics au Ministère de l'Equipement, précise $M$. le Président, a lancé, il y a quelques mois, une étude sur cette question. On a la conviction que les stocks, à l'amont de Nantes, sont beaucoup moins importants qu'ils étaient autrefois; de plus en plus, on est obligé de se tourner vers des sables d'origine marine, c'est-à-dire des sables qu'il faut aller chercher très en aval. 11 y a donc un problème réel dans ce secteur1 à.

M. BALLADE intervient ensuite en ces termes:

A propos de la Seine maritime, le Président Chapon a fait remarquer que les interventions humaines étajent souvent à très faible échelle à côté des phénomènes naturels. C'est certainement très frappant pour des estuaires comme ceux de la Seine ou de la Garonne.

Au contraire, j'ai toujours en l'impression que, dans la Loire, les phénomènes de transfert de sable comme l'importance de «bouchons vaseux», étaient à notre échelle et que les interventions humaines avaient une importance considérable; je parle pour le secteur compris entre Saint-Nazaire et Nantes. Il me semble que tout ce qu'on y fait a des répercussions notables.

Une amélioration par dragage, ou une opération de ce genre, me semble avoir une répercussion très directe sur le comportement du fleuve, sur le comportement des fonds, et sur le façonnement des accès au port de Nantes.

Est-ce que M. MAQUeT partage cette opinion?

Si lon s'en tient aux travaux faits pour l'approfondissement du chenal dans l'ensemble de l'estuaire, répond M. MAQuer, ils paraissent n'apporter qu'une modification mineure au profil en travers. Mais, eu égard aux faibles quantités de sable charriées par la Loire, notre action est effectivement plus à la taillo des problèmes posés que dans les autres estuaires; les quantités de sable qui se déplacent dans la Gironde et dans la Seine sont certainement beaucoup plus importantes. Je ne saurais en dire autant pour le "bouchon vaseux,», phénomène encore mal maîtrisé.

M. le Président clôt la discussion et donne la parole à M. Leror pour l'exposé de la communication qu'il a établi en collaboration avec M. G. BESNIER. 